\title{
Detection of Osteogenesis in Explanted Synthetic Hydroxyapatite-Silicone Orbital Implants Using 3D X-Ray Microscopy
}

\author{
Michael Hahn ${ }^{1}$, Hrishikesh Bale ${ }^{2}$, Leah Lavery ${ }^{2}$ and Björn Busse ${ }^{1}$ \\ ${ }^{1 .}$ Department of Osteology and Biomechanics, University Medical Center Hamburg-Eppendorf, \\ Hamburg, Germany. \\ 2. Carl Zeiss X-ray Microscopy Inc., Pleasanton, CA
}

Hydroxyapatite orbital implants are widely used in the treatment of post-nucleation socket syndrome. The porous hydroxyapatite serves as a matrix for vascular tissue ingrowth. We investigate the degree of fibro-vascular tissue integration and the presence of osteogenesis within the porous hydroxyapatite part of the orbital implants. For comprehensive imaging, all implants were prepared using a grinding technique that allows light microscopy, backscattered electron imaging and 3D X-ray microscopy on undecalcified specimen.

Fibro-vascular integration was present at various degrees in the implants. Pore-diameters in the hydroxyapatite part were measured with 3D X-ray microscopy. Within the implant specimen ossification was detected adjacent to the hydroxyapatite pores. Larger pore diameters were associated with osseous infilling and deep penetration of connective tissue. Specimens with smaller pore diameters presented ossification rather on the surface of the hydroxyapatite implant, whereas those specimens with larger connecting cavities, bone formation was also evident in the center of the implants. Fibro-vascular ingrowth was already found after 3 months implantation period. The 3D X-ray microscopy results indicate that bone formation is induced in the middle of the implant and spreads to the surface.

Clearly, bone formation in the hydroxyapatite part strongly resembles ossification in phthisical eyes. In both entities the neo-ossification shows a very well defined ultrastructure. Several theories exist about the nature of ossification processes, like the hydroxyapatite itself has an osteoinductive effect or myofibroblast from the adjacent extraocular muscles give rise to ossification. Using a 3D-microscopy approach, bone formation in the orbital cavity was found to represent a curious phenomenon due to the fact that there is no connection between the bony tissue and mechanical loading, which could trigger ossification in phthisical eyes or in the center of implants. Therefore, the orbital cavity seems to provide an ideal environment for neo-ossification processes.

References:

[1] Adenis JP et al, Orbit 2003 Sep;22(3):183-91

[2] Sekundo W, Seifert P. Ophthalmology 1998 Mar;105(3):539-43

[3] Shankar A, Surg Neurol. 2002 Dec; 58(6):421-3

[4] Shields CL et al. Ophthalmology 2007 Feb; 114(2):367-73 


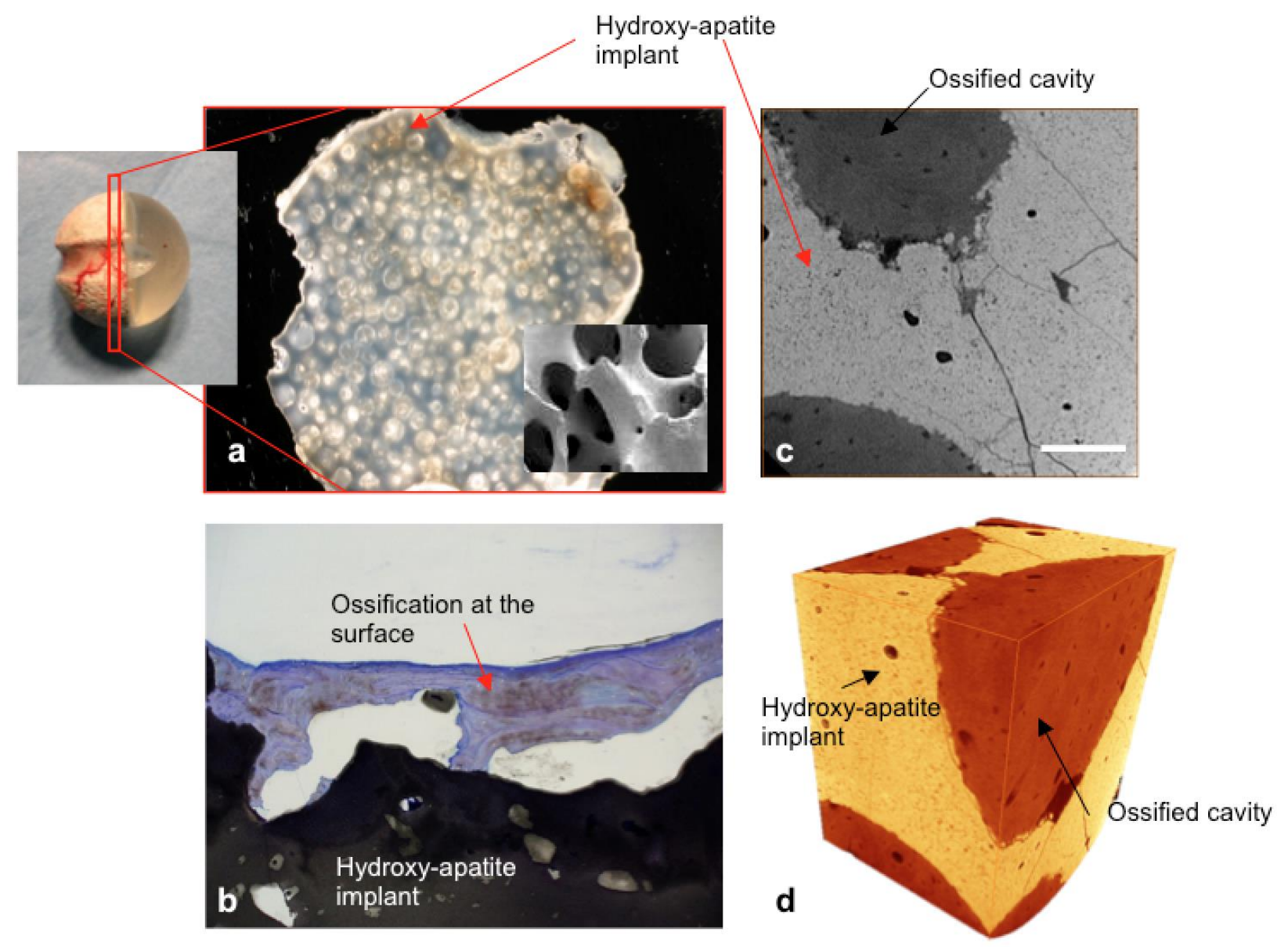

Figure 1. In implants with large connecting pores within the hydroxyapatite part (a), osteogenesis can be detected in toluidine blue stained histology (b), whereas 3D X-ray microscopy (c,d) reveals the penetration depth of the tissue and the preferred zones of ossification in relation to pore diameters. Scale bar corresponds to $100 \mu \mathrm{m}$ 